\title{
Modelling of Dropwise Evaporative Cooling on a Semi-infinite Solid Subjected to Radiant Heat Input
}

\author{
G. WHITE, S. TINKER and M. DI MARZO \\ Mechanical Engineering Department \\ University of Maryland \\ College Park, MD 20742 USA
}

\begin{abstract}
A model for the prediction of dropwise evaporative cooling over hot solid surfaces is proposed for the case of radiant heat input. A detailed representation of the droplet shape during the transient is provided. The direct radiant contribution to the evaporative process is expressed as a liquid-vapor interfacial term and a constant heat absorption term within the liquid layer. The liquid layer is treated with a onedimensional heat conduction approximation justified by previous results and three submodels are used to describe it during the transient. A boundary element method for the solid thermal behavior, previously developed, is extended to this case. The results obtained from a closed-form solution, with simplified solid-liquid interfacial boundary conditions, are also included. Comparisons with the experimental data illustrate the adequacy of the model and the performance of the closed-form solution.
\end{abstract}

KEYWORDS: cooling, evaporation, drops.

\section{NOMENCLATURE}

$\begin{array}{ll}\text { A,B } & \text { constants } \\ \mathrm{c} & \text { specific heat } \\ \mathrm{E}_{\mathrm{b}, \lambda} & \text { blackbody spectral hemispherical } \\ \mathrm{f}_{\phi} & \text { emissive power } \\ \mathrm{F} & \text { ractional surface area coverage } \\ & \text { liquid-vapor interface } \\ \mathrm{h} & \text { overall heat transfer coefficient }\end{array}$

$h_{c}$ convective heat transfer coefficient

$\mathrm{H}$ radiant volumetric heat absorption in the water layer

$\mathrm{J}_{0}, \mathrm{~J}_{1}$ Bessel's functions

$\mathrm{k}$ thermal conductivity

Le Lewis number

n normal to the liquid-vapor interface 
$\mathrm{q}_{\mathrm{o}}$ initial steady state heat flux through the solid

$\mathrm{q}_{\mathrm{c}} \quad$ average heat flux at the solidliquid interface: see Eqs. $(4,5)$

$\mathrm{q}_{\mathrm{r}} \quad$ heat flux by direct radiation

r coordinate: see Fig. 1

$\mathbf{R}$ radius of the wetted region under the droplet

$t$ time

$\mathrm{T}$ temperature

$V$ initial droplet volume

$\mathrm{x} \quad$ vapor molar fraction

$z \quad$ coordinate: see Fig. 1

$\alpha \quad$ thermal diffusivity

$\beta \quad$ shape parameter: $R /(3 V / 4 \pi)^{1 / 3}$

$\delta$ droplet thickness

$\theta$ liquid-vapor-solid contact angle $\boldsymbol{\kappa}_{\lambda} \quad$ water absorption coefficient

$\Lambda \quad$ latent heat of vaporization

$\lambda$ wave length or dummy variable

$\mu \quad$ direction cosine in the water

$\rho_{\phi} \quad$ water air reflectivity

$\tau$ total evaporation time

$\phi$ polar angle

\section{Subscripts}

$\begin{array}{ll}\text { a } & \text { air or far field } \\ \mathrm{i} & \text { liquid-vapor interface } \\ \mathrm{l} & \text { liquid } \\ \mathrm{o} & \text { initial condition } \\ \mathrm{r} & \text { receding condition } \\ \mathrm{s} & \text { solid or at the solid-liquid } \\ & \text { interface }\end{array}$

\section{INTRODUCTION}

Cooling of hot surfaces by droplet sprays has been the subject of numerous investigations. Early experiments of spray cooling by Toda [1] and Bonacina [2] provided insight in the effectiveness of this technique. Detailed studies of single droplets evaporating over hot surfaces heated from below were reported among others by Inada [3], Makino [4] and Takano [5]. In an attempt to extend this information to the fire safety field, a set of dropwise evaporative cooling experiments were performed with radiant heat sources from above the solid surface. DiMarzo [6] investigated single droplets behavior while Dawson [7] provided information on multi-droplet arrays.

Models are proposed for single and multi-droplet systems by a number of investigators. Early models by Seki [8] and diMarzo [9] assumed a constant liquid-vapor interfacial temperature set at an arbitrary value dictated by the solution for two semi-infinite solids brought into sudden contact. This assumption allowed a decoupled treatment of the solid and the liquid. The results of these models are reasonable only for high thermal conductivity solids. More recently, models by Tio [10] and by diMarzo [11] have coupled the liquid and the vapor behavior, thus providing adequate predictions for all non-porous solid materials. However, both these models are for heat input by conduction from below the solid.

This paper addresses the modelling of the droplet evaporative process with radiant heat input from above the solid surface. The model makes use of some of the techniques previously developed by diMarzo [11] and of some of the results obtained for that case. 


\section{PHENOMENOLOGY}

The initial temperature and thermal properties of the solid surface determine how the dropwise evaporation will occur. Evaporation, nucleate boiling and film boiling are the three possible modes observed. This modelling effort is limited to the evaporative mode which is observed at relatively low initial surface temperatures.

A brief overview of the phenomena is provided to explain the rationale for the various assumptions used in the derivation of the model. In the liquid droplet, heat is transferred by conduction since negligible convective motion is observed during the transient [12]. Two major remarks should be made for the specific case of radiant heat input from above the solid surface: a) the droplet vaporization is due in part to the direct radiant input and $b$ ) the droplet shape is greatly affected by direct radiation. These two observations are presented and discussed in detail by diMarzo [6]. The first observation is expected, and the contribution of the direct radiation to the evaporative process will be quantified. The second issue is more subtle since the droplet configuration has a very important impact on its evaporation. In the following, it will be shown that relatively low-frequency direct radiation from above is mostly absorbed in a very thin liquid layer at the liquid-vapor interface. This heat input has a strong effect in relaxing the liquid surface tension thus allowing the droplet to spread on the surface. The consequent increase of the wetted region and the thinning of the liquid layer are great contributors to the enhancement of the heat transfer between the solid-liquid interface and the liquid-vapor interface where evaporation takes place.

A last important consideration must be made concerning the solid thermal behavior. When the heat input is supplied by conduction through the solid, the temperature increases with depth in the normal direction to the solid surface. For the radiant heat input case, the maximum temperature of the solid is at its exposed surface and the temperature decreases with depth. Therefore, an evaporating droplet increases the heat flux toward the wetted region for the conduction case while it decreases the flux for the radiation case. This consideration is very important since it implies that, during the radiant transient, the contribution to the droplet evaporation due to the heat input from below the droplet is depressed. The enhanced heat transfer through the thinner droplet and the direct radiation oppose this effect and the overall evaporation time depends on the combination of all these compensating effects.

\section{MODEL FORMULATION}

\section{Droplet Transient Configuration}

One of the most relevant input parameters to the model is the description of the transient droplet configuration on the solid surface. Two seminal contributions by Chandra [13] and by Zhang [14] illustrate the droplet behavior at the beginning and at the end of the evaporative process. Based on this information, the original assumption (common to all the previous models) of a droplet which has a segment of a sphere geometry for the duration of the evaporative transient must be revised. This 


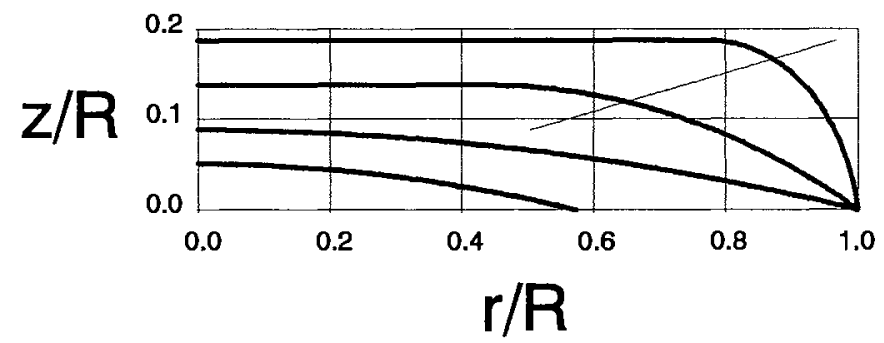

FIGURE 1 Modelled transient geometrical configuration of the droplet.

configuration is fully characterized by a single shape parameter $\beta$ (defined by Bonacina [2] as the ratio of the radius of the wetted area under the deposited droplet and of the equivalent spherical radius of the droplet prior to deposition). This representation needs to be updated, especially in the early portion of the transient, in light of the evidence compiled by Chandra [13] which clearly demonstrated that a flattened shape exists after deposition. This modification of the droplet shape configuration requires the introduction of a second parameter, namely the liquid-vapor-solid contact angle $\theta$.

The resulting shape with these two parameters has been characterized by considering the value of the parameter $\beta$ at deposition and the values of the parameter $\theta$ at deposition and at the onset of the shrinkage of the wetted region which occurs when $\theta$ reaches its receding value. The receding angle, $\theta_{r}$, is defined as the minimum liquid-vapor-solid contact angle consistent with the balance of the surface tension and surface adhesion forces. When the contact angle reaches this limiting value, additional reduction in the droplet volume will cause shrinkage of the wetted surface.

The first assumption in modelling the transient droplet configuration is that the droplet shape can be characterized as a segment of a sphere once $\theta$ reaches the receding value. This assumption is reasonable since the surface tension must first reconfigure the liquidvapor interface (to minimize its surface area) before it can shrink the solid-liquid interface. The initial value of the parameter $\beta_{\mathrm{o}}$ identifies the radius (i.e., the area) of the solid-liquid interface up to the receding conditions. Therefore, the value of $\theta_{\mathrm{r}}$ (given the solid-liquid interfacial area) identifies a unique volume of liquid at the receding conditions. This droplet configuration is used as a milestone in the transient configuration since, for any subsequent times, the droplet will retain the same aspect ratio; thus, it is fully characterized.

The second assumption in the modelling of the transient droplet configuration is needed to unequivocally define the gradual transition from the initial configuration to the configuration at the receding conditions. This assumption states that the droplet apex will always be less or equal to its initial value. Therefore, the droplet apex at the receding condition constitutes a minimum value for the initial value of the droplet apex and thus a maximum bound for the angle $\theta_{\mathrm{o}}$. Note that another upper bound of $\theta_{\mathrm{o}}$ is obtained by Chandra [13] at $90^{\circ}$ for evaporating and boiling droplets (nucleate boiling). 


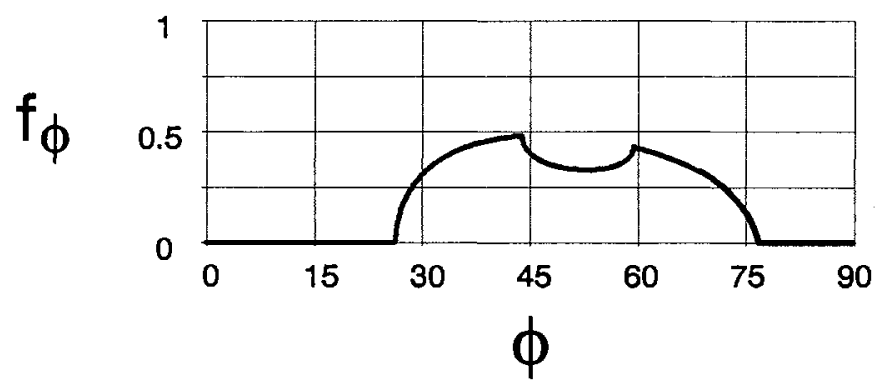

FIGURE 2 Fractional surface area coverage (data from [6]).

The minimum value of the angle $\theta_{0}$ is obtained if a spherical segment configuration is assumed at deposition. To unequivocally set the initial droplet configuration, one may want to neglect the small recoiling effect described by Chandra [13] and conclude that

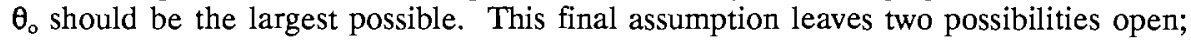
the value of $\theta_{0}$ is equal to the smaller of: a) its maximum bound set by the value of the droplet apex at the receding conditions or b) $\theta_{0}=90^{\circ}$. Note that, for the second case, it follows that the droplet apex at deposition is larger than at the receding conditions.

Consider the intersection of the tangents to the droplet liquid-vapor interface at the droplet apex and at the liquid-vapor-solid contact point. One can identify such an intersection point at deposition and a similar point at the receding conditions. For case a) a straight line connecting these two points is horizontal while for case b) it is depicted in Fig. 1. By constraining the intercept of the two tangents to be on this line, one obtains a condition that insures a gradual change in the droplet geometrical configuration which is reasonably consistent with the experimental observations [13]. Figure 1 provides a set of geometrical configurations that illustrate the typical results of this model.

\section{Direct Radiation in the Liquid Layer}

A significant effort has been devolved to capture the main features of the direct radiation contribution to the water droplet evaporation while retaining a simple approach amenable to the model formulation. The first step is to characterize the specific radiant source. For the data obtained by diMarzo [6], the geometry of the radiant heat source has been described in Fig. 2 where two electric radiant panels are located above the surface and are identified in terms of their respective fractional surface area coverage at various polar angles $\phi$ above the solid surface. The following assumptions are made: a) the radiant panels behave as black bodies; b) the radiation scattering within the water droplet is negligible; c) the liquid-vapor interface is horizontal and flat; and d) the radiation reaching the liquid-solid interface is completely absorbed by the solid. The volumetric heat absorption in the liquid layer is given as: 


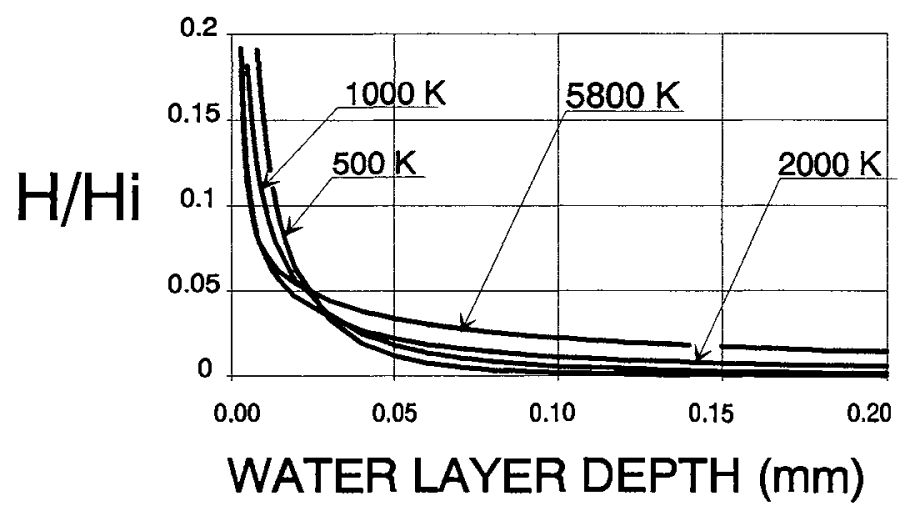

FIGURE 3 Normalized heat absorption in the liquid due to direct radiation (data from [6]).

$$
H=2 \int_{0}^{\infty} E_{b, \lambda} \kappa_{\lambda} \int_{0}^{\pi / 2} \frac{1}{\mu} f_{\phi} \cos \phi \sin \phi\left(1-\rho_{\phi}\right) e^{-\frac{\kappa_{\lambda}(\delta-z)}{\mu}} d \phi d \lambda
$$

The absorption coefficient $\kappa_{\lambda}$ is a very strong function of the wave length $\lambda$, the direction cosine $\mu$ is given by the Snell's law, the fractional surface area coverage $f_{\phi}$ is obtained from Fig. 2, and the reflectivity $\rho_{\phi}$ is less than 0.1 for $\phi$ less than $65^{\circ}$ and is given by the electromagnetic theory. The direct radiation into the water layer for the geometrical configuration used by diMarzo [6] is shown in Fig. 3. The volumetric heat absorption is high in a thin layer near the liquid-vapor interface (consider a layer thickness of about $0.05 \mathrm{~mm}$ ). This is true over a broad range of the radiant surface temperature. Therefore, one can split the direct radiation in three parts: a) an interfacial flux term $F$ (which is the integral of $H$ over the thin layer thickness); b) a volumetric heat absorption term $H$ which can be considered a constant, uniformly distributed heat source throughout the liquid layer; and c) a residual term which accounts for the incoming radiation at the solid-liquid interface. This last term is evaluated from an energy balance by deducting from the incoming radiant flux at the liquid-vapor interface the two previous terms.

Careful consideration must be given to the fact that the liquid-vapor interface is not flat. The flat surface assumption is useful to obtain simple results as shown in Fig. 3. However, a significant error can be introduced in the evaluation of the total incoming radiation when significant radiant surfaces are present at large polar angles $\left(\phi>60^{\circ}\right)$. To rectify this problem, a multiplier must be introduced which accounts for the liquidvapor interface orientation given the transient geometrical configuration of the droplet. 
The results of the coupled model proposed by diMarzo [11] show that the heat transfer by conduction in the liquid layer is mostly one-dimensional in the direction normal to the solid-liquid interface. This is true everywhere and at all times with the exception of the region in the immediate proximity of the droplet edge where the radial component of the flux may be as large as ten percent of the total flux. The thinner geometrical configuration of the droplet subjected to the direct radiant field reinforces the assumption that the liquid may be treated as a one-dimensional conduction medium with generation (uniform internal generation is used to account for the residual contribution of the direct radiation not absorbed in the proximity of the liquid-vapor interface).

The one-dimensional modeling of the transient heat conduction in the liquid region encompasses three sub-models: a) initial contact closed-form solution; b) full transient diffusion equation; and c) quasi-steady state conduction equation. In the early portion of the transient, the liquid layer behaves as a semi-infinite solid while the heat wave propagates through its thickness. The solution of this problem is the classical solution for two semi-infinite solids (initially at different temperatures) brought into sudden contact [15]. Note that this solution is valid for a very short time (fraction of a second) especially where the liquid layer is thin (i.e. at the droplet edge). The relevance of this sub-model is to provide a smooth temperature profile in the liquid and a heat flux at the solid-liquid interface to initiate the numerical computations.

When the heat wave through the liquid reaches the liquid-vapor interface, the full transient diffusion equation sub-model is used. The diffusion equation written for the liquid yields a tri-diagonal matrix solution (with the one-dimensional heat flux approximation used here). At the time when this full transient solution is first used, the temperature profile is given by the closed-form solution for the two semi-infinite solids brought into sudden contact. At any subsequent time, the temperature at $z=0$ is given by the solution of the semi-infinite solid which will be discussed in the following. The liquid-vapor boundary condition at $z=\delta$ (where $\delta(r)$ is given by the transient droplet configuration model) can be written as [11]:

$$
-k_{l} \nabla T \cdot \hat{n}=0.62\left(\frac{h_{c} \Lambda}{c_{a} L e^{2 / 3}}\right) \frac{x_{i}-x_{a}}{1-x_{i}}+h\left(T_{i}-T_{a}\right)-F
$$

Note the term $F$ which describes the direct radiation contribution absorbed near the liquid-vapor interface. This term is configured as an interfacial heat flux since the layer thickness of $0.05 \mathrm{~mm}$ (see Fig. 3 ) is much smaller than the computational grid size.

When the liquid heat capacity term becomes small (i.e: the transient solution and the quasi-steady state solution are within less than 3 percent), the quasi-steady state conduction equation sub-model takes over. This third sub-model provides a very fast solution for the liquid layer. At the solid-liquid interface, the quasi-steady state conduction equation (with the constant heat source term discussed previously) yields a heat flux given by: 


$$
-k_{s} \frac{\partial T}{\partial z}=\frac{k_{l}}{k_{l}+A \delta}\left(\frac{A H \delta^{2}}{2 k_{l}}+H \delta-A T_{s}-B\right)
$$

The vapor-liquid boundary condition on the right hand side of Eq. (3) is linearized as a function of the interfacial temperature $T_{\mathrm{i}}$ (i.e.: $A T_{\mathrm{i}}+B$ ). Note that the vapor molar fraction at the liquid-vapor interface is a function of the interfacial temperature. By expanding the boundary condition in a two-term series and by making use of the Clausius-Clapeyron relationship to differentiate the molar fraction with respect to the interfacial temperature, $A$ and $B$ are obtained analytically.

The coupling of these solutions for the liquid region with the solid solution is done with a simple predict-correct method where the heat flux distribution is the input to the solid solution and the interfacial solid-liquid temperature distribution is the output. To this effect, note the formulation of Eq. (3) which readily provides the heat flux at the solid surface as a function of the interfacial temperature $T_{\mathrm{s}}$.

To provide a general indication of the role played by the three sub-models, consider that: a) the first sub-model is applicable for a very short time at the initiation of the transient; b) the full transient sub-model is used for about sixty per cent of the transient thereafter; and c) the quasi-steady state sub-model takes over for the last forty percent of the transient.

\section{Modelling of the Semi-Infinite Solid}

The solid thermal behavior is described by the transient two-dimensional $(r, z)$ diffusion equation. The boundary conditions provide full coupling at the solid-liquid interface and state that the droplet effect is negligible in the far field. The solution of this equation is obtained with a boundary element method previously used for the conduction case [11]. The details of this method have been presented in the cited reference and in a number of previous publications.

\section{Closed-Form Solution}

In addition to the previous model, a closed-form solution for the transient surface temperature distribution is presented. By assuming that the solid-liquid interfacial heat flux is constant and uniform, one can obtain the following expression [15]:

$$
T_{s, 0}-T=\frac{\left(q_{c}-q_{o}\right) R}{h_{s}} \int_{0}^{\infty} J_{0}(\lambda r) J_{1}(\lambda R) \operatorname{erf}\left(\lambda \sqrt{\alpha_{s} t}\right) \frac{d \lambda}{\lambda}
$$

This form is used during the evaporation transient (i.e., $t<\tau$ ). Thereafter, the following modified form is used: 


$$
T_{s, 0}-T=\frac{\left(q_{c}-q_{o}\right) R}{k_{s}} \int_{0}^{\infty} J_{0}(\lambda r) J_{1}(\lambda R)\left\{\operatorname{erf}\left(\lambda \sqrt{\alpha_{s} t}\right)-\operatorname{erf}\left[\lambda \sqrt{\alpha_{s}(t-\tau)}\right]\right\} \frac{d \lambda}{\lambda}
$$

This solution is in good agreement with the experimental data and the numerical computations for the conduction case [11]. For the case under consideration here, the closed-form solution refers to the heat associated with the droplet volume vaporized by conduction. The portion of the liquid vaporized by direct radiation will be excluded from the term $q_{\mathrm{c}}$ which represents the average heat flux transferred to the droplet during the whole evaporative transient through the solid-liquid interface (i.e., $\pi R_{\mathrm{o}}{ }^{2}$ ). Note that this solution requires as input the total evaporation time $\tau$ as well as the fraction of the vaporization heat input due to direct radiation which are outputs of the previous model.

\section{MODEL VALIDATION}

The results of the model are compared with the experimental findings of diMarzo [6]. The measured and calculated total evaporation times are within less than 10 percent (which is the scatter of the experimental data).

The surface temperature distribution of the model is compared with the experimental measurements [6] in Fig. 4. The data are for two initial solid surface temperatures and for the same droplet volume. Some discrepancies are observed during the transient after the droplet evaporation. However, as time passes (i.e., $t>1.3 \tau$ ), they tend to disappear.

Overall, the model predictions are in good agreement with the data. Additionally, the temperature distributions obtained with the closed-form solution are also plotted (dashed lines) to show that Eqs. (4) and (5) provide a reasonable representation of the phenomena. The total evaporation time and the evaporative component due to the direct radiation must be known in advance to use the closed-form solution. The relevance of this solution is for its use in the formulation of multi-droplet models for the prediction of the performance of sparse sprays. Therefore, the closed-form solution should be regarded as a useful fitting function to concisely represent the transient surface temperatures.

Note that the infrared thermography, used to acquire the data, is unable to provide data for the temperature at the solid-liquid interface. It is interesting to note the different behavior of the closed-form solution and of the model in the wetted region. The results are consistent with the different boundary conditions (i.e., uniform flux for the closedform and a coupled liquid-solid condition for the model).

\section{DISCUSSION}

The validated model can be used to gain additional insight into the evaporative transient phenomena. One aspect worth considering is the effect of the initial value of the liquidgas-solid contact angle. As pointed out previously, there is a range of values which is bounded by a flattened shape and a spherical cap. The first question concerns the 


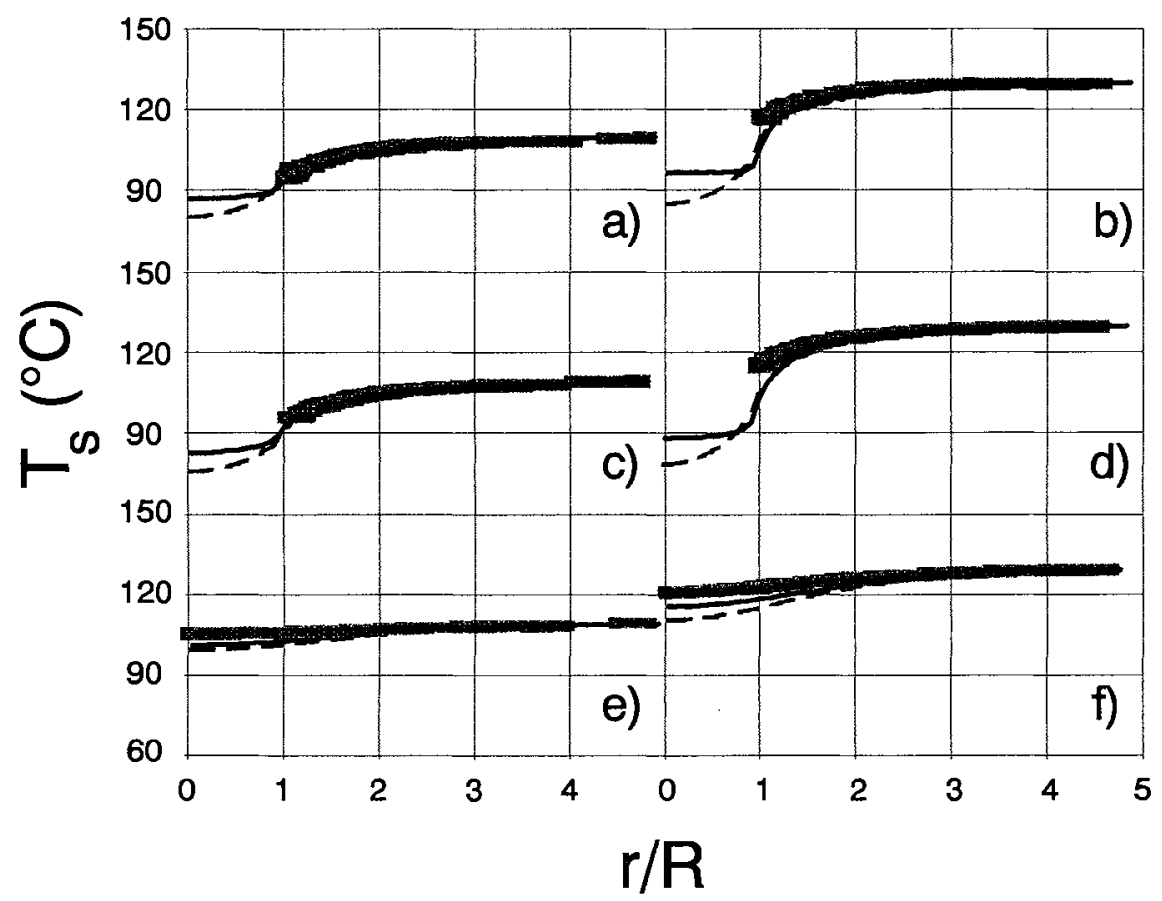

FIGURE 4 Model and closed-form solution validation (V = $10 \mu \mathrm{l}$ ). Data (from [6]): shaded; model: solid line; closed-form: dashed line. a),c) and $\mathrm{e}$ ): $\mathrm{T}_{\mathrm{s}, \mathrm{o}}=110^{\circ} \mathrm{C}$ at $0.3,0.9$ and $1.1 \mathrm{t} / \tau$. b), d) and $\mathrm{f}$ ): $\mathrm{T}_{\mathrm{s}, \mathrm{o}}=130^{\circ} \mathrm{C}$ at $0.3,0.9$ and $1.1 \mathrm{t} / \tau$.

sensitivity of the results with the initial value of the contact angle. The model indicates that little effect is observed in the overall evaporation time. Figure 5 illustrates the transient behavior of $\beta$, of the ratio $R / R_{o}$ (which characterizes the shrinkage of the wetted region) and of $\theta$ for the maximum and minimum initial values of this parameter. Note that the uniqueness of the value at the receding conditions and the invariance of the total evaporation time with $\theta_{0}$ is reflected in the independence of the shape parameter and radius of the wetted region from the initial value of the contact angle. Chandra [13] reports values of the contact angle at deposition between $32^{\circ}$ and $90^{\circ}$ for conditions similar to the one reported here. The data from diMarzo [6] are in reasonable agreement with the model estimates.

Another relevant aspect is the direct radiation contribution to the evaporative process which has been discussed previously. According to the model computations, the evaporative component by direct radiation grows more slowly than the solid-liquid interfacial flux with increasing initial solid surface temperatures. Table 1 summarizes these findings and also provides the total evaporation time for the various cases. 


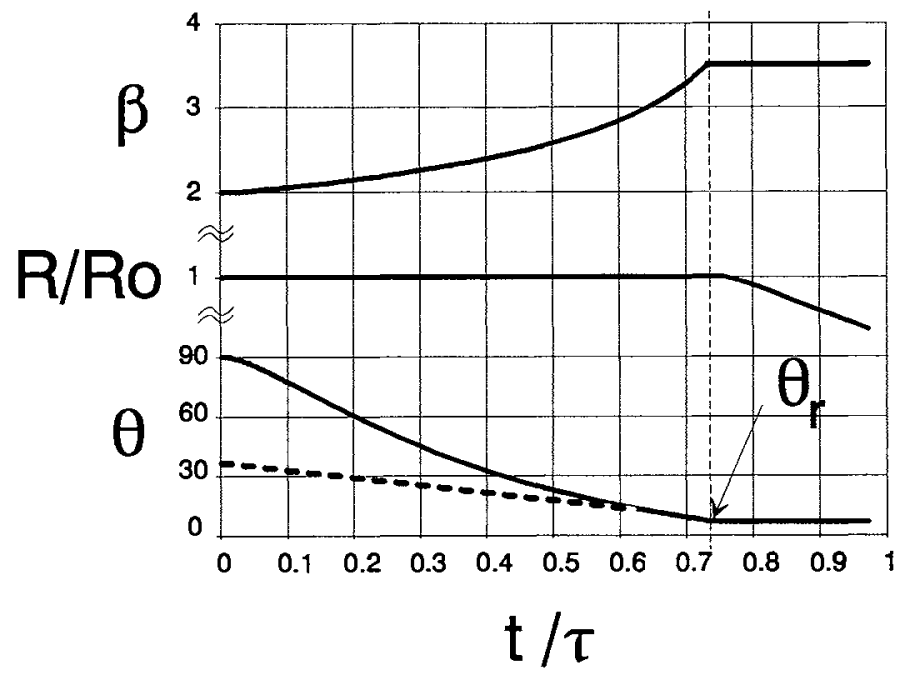

FIGURE 5 Transient behavior of the parameters governing the droplet shape $\left(T_{\mathrm{s}, \mathrm{o}}=130\right.$ $\left.{ }^{\circ} \mathrm{C} ; \mathrm{V}=10 \mu \mathrm{l}\right) \theta_{\mathrm{o}}$ maximum: solid line; $\theta_{\mathrm{o}}$ minimum: dashed line

TABLE 1 Evaporation time, radiant and conductive heat fluxes for various $T_{s, o}$

\begin{tabular}{|c|c|c|c|}
\hline $\mathrm{T}_{\mathrm{s}, \mathrm{o}}\left[{ }^{\circ} \mathrm{C}\right]$ & $\tau[\mathrm{s}]$ & $\mathrm{q}_{\mathrm{c}}\left[\mathrm{kW} / \mathrm{m}^{2}\right]$ & $\mathrm{q}_{\mathrm{r}}\left[\mathrm{kW} / \mathrm{m}^{2}\right]$ \\
\hline 90 & 103 & 5.0 & 5.5 \\
\hline 100 & 78 & 9.1 & 5.8 \\
\hline 110 & 58 & 12.6 & 6.1 \\
\hline 120 & 46 & 17.3 & 6.5 \\
\hline 130 & 37 & 22.8 & 6.9 \\
\hline 140 & 31 & 28.4 & 7.2 \\
\hline
\end{tabular}

\section{CONCLUSIONS}

A model has been presented which predicts the transient thermal behavior of the coupled droplet-solid system for evaporative cooling due to radiant heat input. The transient droplet shape is modeled introducing a shape parameter and the liquid-gassolid contact angle. The model predicts the total evaporation time and the transient temperature distribution over the solid surface. A closed-form solution is suggested as a simple fitting routine to represent the solid surface thermal behavior. The closed-form solution and the model results are in good agreement with the experimental data. The closed-form solution will be used to represent the solid surface temperature distribution 
in multi-droplet models which simulate the cooling effect of sparse sprays.

\section{ACKNOWLEDGEMENTS}

This study has been supported by the Building and Fire Research Laboratory of the National Institute of Standards and Technology. The authors wish to express their gratitude to Drs. Baum and Evans for their guidance and encouragement.

\section{REFERENCES}

1. Toda, S., "A Study of Mist Cooling. First Report: Investigation of Mist Cooling," Heat Transfer, Japanese Research, 1: 3, 39-50, 1972.

2. Bonacina, C., Del Giudice, S. and Comini, G., "Dropwise Evaporation," Transactions ASME, Journal of Heat Transfer, 101: 441-446, 1979.

3. Inada, S., Mikasaka, Y. and Nishida, K., "Transient Heat Transfer for a Water Drop Impinging on a Heated Surface," Bulletin of JSME, 28: 246, 2675-2681, 1985.

4. Makino, K. and Michiyoshi, I., "The Behavior of a Water Droplet on Heated Surfaces," International Journal of Heat and Mass Transfer, 27: 781-791, 1984.

5. Takano, T. and Kobayasi, K., "Vaporization Behavior of a Single Droplet Impinging on a Heated Surface With a Flame-Sprayed Ceramic Coating," Heat Transfer, Japanese Research, 20: 1-17, 1991.

6. diMarzo, M., Kidder, C.H. and Tartarini, P., "Infrared Thermography of Dropwise Evaporative Cooling of a Semi-Infinite Solid Subjected to Radiant Heat Input," Experimental Heat Transfer, 5: 101-114, 1992.

7. Dawson, H.F., and diMarzo, M., "Multi-Droplet Evaporative Cooling: Experimental Results," AIChE Symposium Series, 89: 26-35, 1993.

8. Seki, M., Kawamura, H. and Sanokawa, K., "Transient Temperature Profile of a Hot Wall Due to an Impinging Liquid Droplet," Transactions ASME, Journal of Heat Transfer, 100: 167-169, 1978.

9. diMarzo, M. and Evans, D.D., "Evaporation of a Water Droplet Deposited on a Hot High Thermal Conductivity Surface," Transactions ASME, Journal of Heat Transfer, 111: 210-213, 1989.

10. Tio, K.K. and Sadhal, S.S., "Thermal Analysis of Droplet Spray Evaporation from a Heated Solid Surface," Transactions ASME, Journal of Heat Transfer, 114: 220233, 1992.

11. diMarzo, M., Tartarini, P., Liao, Y., Evans, D. and Baum, H., "Evaporative Cooling Due to a Gently Deposited Droplet," International Journal of Heat and Mass Transfer, 36: 4133-4139, 1993.

12. Ostrach, S. and Pradhan, A., "Surface-Tension Induced Convection at Reduced Gravity," AIAA Journal, 16: 5, 419-424, 1978.

13. Chandra, S. and Avedisian, C.T., "On the Collision of a Droplet With a Solid Surface," Proceedings of the Royal Society, 432: 13-41, 1991.

14. Zhang, N. and Yang, W.J., "Natural Convection in Evaporating Minute Drops," Transactions ASME, Journal of Heat Transfer, 104: 656-662, 1982.

15. Carslaw, H.S., and Jaeger, J.C., Conduction of Heat in Solids, Clarendon Press, Oxford, 87-88, 214-216 and 264, 1959. 